\title{
Plan de cuidados al prematuro con síndrome de dificultad respiratoria: fortaleciendo el vínculo madre-recién nacido
}

\author{
Carolina Chinchay Quispe ${ }^{1}$
}

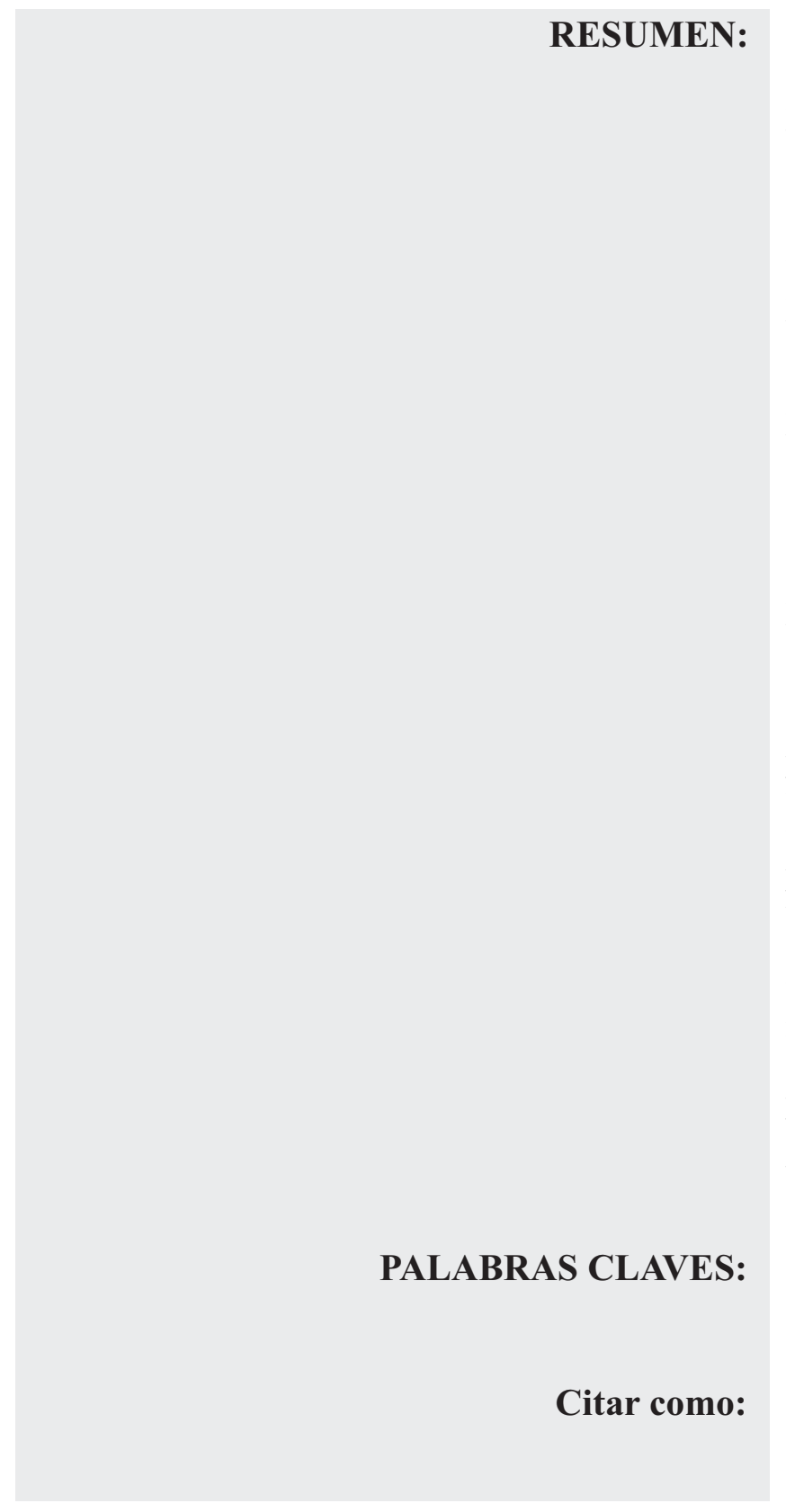

El síndrome de dificultad respiratoria es la principal causa de morbilidad en los recién nacidos prematuros porque en este periodo postnatal se presenta una inmadurez fisiológica y anatómica pulmonar que impide mantener una respiración e intercambio gaseoso adecuados. El presente reporte de caso trata de un recién nacido pretérmino de 36 semanas que presentó un bajo peso al nacer de $2280 \mathrm{~g}$ y una dificultad respiratoria que se evidenció por taquipnea, disnea e hipoxia. Esta inestabilidad fisiológica del prematuro requirió internamiento, situación que conllevó a una interrupción del vínculo afectivo entre mamá-recién nacido. Ante la valoración del caso se decidió hacer una planificación de los cuidados de enfermería con enfoque humanístico, para ello se propusieron cuatro diagnósticos de relevancia: "Patrón respiratorio ineficaz", "Patrón de alimentación ineficaz del lactante", "Ansiedad" y "Riesgo de deterioro de la vinculación". Se ejecutó el plan de cuidados durante los primeros cinco días de vida del recién nacido utilizando el modelo de valoración de los 13 dominios según aspectos positivos y negativos de la Taxonomía II de NANDA-I y el lenguaje estandarizado de los diagnósticos, intervenciones y resultados de la Taxonomía II. Al finalizar el proceso se lograron mejorar los patrones de respiración y alimentación disminuyendo así las complicaciones en la salud del recién nacido prematuro. Asimismo, se alcanzó fortalecer el vínculo entre la mamá y el recién nacido logrando el bienestar biopsicosocial y potenciando el rol materno.

Atención de Enfermería; Prematuro; Síndrome de Dificultad Respiratoria del Recién Nacido; Vínculo.

Chinchay C. Plan de cuidados al prematuro con Síndrome de dificultad respiratoria: fortaleciendo el vínculo madre-recién nacido. CASUS. 2016; 1(1): 41-50. 


\section{INTRODUCCIÓN}

La Organización Mundial de la Salud indica que cada año nacen en el mundo unos 15 millones de recién nacidos prematuros, es decir, más de uno en 10 nacimientos. El prematuro es el bebé nacido antes de las 37 semanas de gestación y tiene un peso al nacer menor de $2,5 \mathrm{~kg}$ y por la inmadurez pulmonar que presenta el $20 \%$ de ellos padecen de patologías respiratorias (1).

Estudios epidemiológicos en un Hospital Materno Infantil de México y en 5 hospitales del Perú concluyen que el síndrome de dificultad respiratoria (SDR) es la principal causa de morbilidad en los recién nacidos prematuros $(2,3)$. Esta patología se presenta por la inmadurez en el desarrollo fisiológico y anatómico pulmonar del prematuro, cuyo déficit de surfactante impide mantener un intercambio gaseoso adecuado (4).

Todo recién nacido prematuro por su inestabilidad fisiológica requiere ser hospitalizado. Esta situación conlleva a una disrupción del vínculo afectivo entre padres-hijo afectando el desarrollo físico, psicológico e intelectual del hijo y la relación que se desarrollará a lo largo del tiempo entre ambos (5). Así lo señalan estudios realizados en los niños prematuros hospitalizados que no establecieron un contacto con sus padres. En consecuencia presentaron con mayor frecuencia otras morbilidades y alteraciones en el desarrollo neurocognitivo, neurosensorial y conductual. Los estudios concluyeron que el impacto emocional que los padres experimentan al no poder interrelacionarse con su hijo recién nacido repercute en forma negativa en la formación del vínculo y el proceso de crianza (6).

De acuerdo a la problemática mencionada, en el presente reporte de caso se propuso realizar las intervenciones de enfermería al recién nacido prematuro con síndrome de dificultad respiratoria fortaleciendo el vínculo madre-hijo. En la actualidad en numerosas unidades neonatales peruanas se descuida o se desconocen las consecuencias positivas de brindar una atención de enfermería humanizada orientada a fortalecer la formación del vínculo afectivo entre padres e hijo. De este modo, se incumple con el Derecho de los Niños Hospitalizados, respaldado por el marco jurídico internacional y nacional, en donde se señala que todo niño hospitalizado debe estar acompañado por su familia todo el tiempo (7). Para la resolución del caso se empleó el modelo de los dominios de la Taxonomía II de NANDA-I y la clasificación de los diagnósticos, intervenciones y resultados de enfermería de la Universidad de Iowa (NANDA-I, Nursing Interventions Classification (NIC) y Nursing Outcomes Classification (NOC), respectivamente (8-10).

\section{PRESENTACIÓN DEL CASO}

Se presenta el caso de un recién nacido pretérmino adecuado para la edad gestacional (RNPT AEG), que nació por cesárea de emergencia a las 36 semanas de gestación (según test de Capurro) debido a que la mamá ingresó presentando hemorragia en el tercer trimestre del embarazo por desprendimiento prematuro de placenta. Al nacer se le valoró con el test de Apgar 7 al minuto, 9 a los 5 minutos y con el Test de Silverman-Anderson 4 puntos. Presentó bajo peso al nacer (BPN): $2280 \mathrm{~g}$, talla: 45,5 cm, circunferencia cefálica: $32 \mathrm{~cm}$ y circunferencia torácica: $30 \mathrm{~cm}$.

El recién nacido prematuro ingresó a la unidad de neonatología diagnosticado con síndrome de dificultad respiratoria (SDR) presentando taquipnea de 68 respiraciones por minuto, saturación de oxígeno (SO2) de $90 \%$, glicemia de $35 \mathrm{mg} / \mathrm{dl}$ e hipoactividad. Se le instaló en una servocuna con técnica de nido acolchado y terapia de oxígeno que fue administrado a presión positiva continua en la vía aérea nasal mediante sistema burbuja bajo el agua (underwater bubble Continuous Positive Airway Pressure, (CPAP) burbuja bajo el agua), también recibió por vía endovenosa tratamiento de infusión: dextrosa al 10\% para mejorar el nivel de glucemia, se le colocó una sonda orogástrica a gravedad (SOG) y se indicó que no reciba nada por vía oral (NPO) durante los dos primeros días de vida.

Al segundo día de hospitalización ingresó la mamá a ver a su bebé por un periodo corto, manifestó haber experimentado angustia por la salud de su bebé y frustración por no poder cuidarlo.

\section{VALORACIÓN GENERAL}

Para el estudio del caso clínico se realizó la valoración empleando los dominios de la Taxonomía II de NANDA-I enfocándose en la interacción del recién 
nacido y la mamá y se identificaron aspectos negativos a nivel fisiológico en el recién nacido prematuro y también a nivel psicosocial en el afrontamiento del problema y el desempeño de roles del binomio madre e hijo. Para la valoración completa ver Tabla 1.

\section{FASE DIAGNÓSTICA}

Según la valoración realizada al recién nacido y a la mamá se formularon diagnósticos reales y de riesgo utilizando los diagnósticos de enfermería de la Taxonomía II de NANDA - I. Se priorizaron cuatro diagnósticos que permitieron brindar cuidados de enfermería en respuesta a las necesidades biopsicosociales del binomio mamá/recién nacido.

\section{Reales:}

- (00032) Patrón respiratorio ineficaz r/c con inmadurez pulmonar e/p disnea, taquipnea, aleteo nasal, alteración en la profundidad respiratoria, SO2: $90 \%$. Dominio 4, clase 4.

- (00107) Patrón de alimentación ineficaz del lactante $\mathrm{r} / \mathrm{c}$ prematuridad e/p incapacidad para coordinar la succión, la deglución y la respiración. BPN: 2280 g. Dominio 2, clase 1.

- (00146) Ansiedad r/c cambio en la función del rol e/p angustia, temor, preocupación. Dominio 9 , clase 2 .

\section{De riesgo:}

-(00058) Riesgo de deterioro de la vinculación $\mathrm{r} / \mathrm{c}$ la enfermedad del niño impide la iniciación eficaz del contacto parental. Dominio: 7, clase: 2 .

\section{FASE DE PLANIFICACIÓN}

Para la realización del plan de cuidados se fijó el horario de visitas diarias para generar el apego entre la mamá y su hijo fortaleciendo el vínculo afectivo. Durante la estancia de la mamá en la unidad neonatal se le brindó asesoramiento permanente para desarrollar la confianza y competencia necesaria para que desempeñe su rol satisfactoriamente. Para el plan de cuidados ver Tabla 2 .

\section{FASE DE EJECUCIÓN}

Durante los primeros cinco días de vida del recién nacido se llevó a cabo el plan de intervenciones de enfermería destinado a mejorar el desarrollo fisiológico del recién nacido, principalmente la respiración y la nutrición. A partir del tercer día del proceso se integraron los cuidados de la mamá buscando en esta interrelación materno-filial el bienestar biopsicosocial de ambos. Para documentar las actividades de enfermería se utiliza el lenguaje estandarizado de la taxonomía NIC.

\section{FASE DE EVALUACIÓN}

Tal como se evidencia en la tabla 2 hubo una mejoría significativa y positiva en la resolución del caso. En el diagnóstico de Patrón respiratorio ineficaz se logró con las intervenciones de monitorización respiratoria y oxigenoterapia alcanzar el objetivo propuesto de que el recién nacido mejorara el patrón respiratorio ineficaz y en consecuencia el intercambio gaseoso. En concreto el bebé pasó del indicador 2 (desviación sustancial del patrón respiratorio normal) al indicador 4 (desviación leve del patrón normal). Se corroboró este logro con una puntuación menor de tres en la valoración de Silverman- Anderson que indica un cuadro de dificultad respiratoria leve en el recién nacido.

En el diagnóstico Patrón de alimentación ineficaz se consiguió con la alimentación enteral por sonda proporcionar los nutrientes necesarios para cubrir las necesidades metabólicas del bebé, a pesar que durante este periodo de transición de la vida del prematuro no se gane peso. También la monitorización nutricional corroboró que la pérdida de peso era la esperada y estaba en concordancia con la talla y la edad gestacional del bebé. Se incrementó un punto del indicador inicial logrando alcanzar el indicador 4 (desviación leve del estado nutricional adecuado).

Se identificó el diagnóstico Ansiedad basado en el temor, angustia y preocupación de la mamá ante el desempeño de su rol. Por ello mediante las intervenciones de enfermería se fomentó y potenció el rol materno, alcanzando que la mamá disminuyera los sentimientos de frustración que experimentó por la 
separación de su bebé ante el problema de salud y desempeñara su rol con seguridad y satisfacción. El resultado fue que se incrementaron 2 puntos del indicador inicial logrando el indicador 4 (frecuentemente demostró mejorar el rol maternal).
En cuanto al diagnóstico Riesgo de deterioro de la vinculación se disminuyó fomentando el apego de la mamá y el recién nacido restableciendo los lazos de seguridad y confianza entre ambos. El indicador inicial mejoró en 2 puntos y se obtuvo al final de la intervención el indicador 4 (levemente comprometidos los lazos afectivos).

Tabla 1. Análisis de los Dominios, según aspectos positivos y negativos

\begin{tabular}{|c|c|c|}
\hline Dominios & Positivos & Negativos \\
\hline Promoción de la salud & -No se observa & $\begin{array}{l}\text { - Gestante llegó al hospital con } \\
\text { hemorragia en el tercer trimestre } \\
\text { por desprendimiento prematuro } \\
\text { de placenta } \\
\text {-RNPT AEG, nació mediante } \\
\text { cesárea de emergencia a las } 36 \\
\text { semanas de gestación (según test } \\
\text { de Capurro) } \\
\text {-RNPT presentó bajo peso al } \\
\text { nacer: } 2280 \mathrm{~g} \text {. }\end{array}$ \\
\hline Nutrición & $\begin{array}{l}\text { - Recibió por vía endovenosa } \\
\text { dextrosa al } 10 \% \text { con velocidad de } \\
\text { infusión de } 5 \mathrm{mg} / \mathrm{Kg} / \text { minuto. } \\
\text {-Al tercer día de hospitalización } \\
\text { inició nutrición enteral por sonda. }\end{array}$ & $\begin{array}{l}\text {-PortadordeSOG. } \\
\text {-Débil reflejo de succión y } \\
\text { deglución. }\end{array}$ \\
\hline Eliminación e Intercambio & $\begin{array}{l}\text { •Micción espontánea } \\
\text { •Presencia de meconio }\end{array}$ & $\begin{array}{l}\text {-RNPT presentó taquipnea de } 68 \\
\text { respiraciones por minuto, SO } 2 \text { de } \\
90 \% \text {, Test de Silverman- } \\
\text { Anderson } 4 \text { puntos. } \\
\text { - Recibió oxigenoterapia CPAP } \\
\text { burbuja. }\end{array}$ \\
\hline Actividad / Reposo & -No se observa & $\begin{array}{l}\text {-Sueñoligero y agitado. } \\
\text {-Hipoactivo, sólo presenta } \\
\text { movimientos defensivos ante } \\
\text { estímulo doloroso. } \\
\text {-Recibió oxigenoterapia CPAP } \\
\text { burbuja. }\end{array}$ \\
\hline
\end{tabular}




\begin{tabular}{|c|c|c|}
\hline Dominios & Positivos & Negativos \\
\hline Percepción / Cognición & $\begin{array}{l}\text {-Presentó llanto vigoroso } \\
\text { sólo al ejecutar procedimientos } \\
\text { dolorosos }\end{array}$ & $\begin{array}{l}\text { - La mamá desconocía cómo } \\
\text { manipular a su bebé que se } \\
\text { encontraba en una incubadora } \\
\text { conectadoa varios dispositivos. }\end{array}$ \\
\hline Autopercepción & -No se observa & $\begin{array}{l}\text { - La mamá percibe que no ha } \\
\text { podido realizarse comotal. }\end{array}$ \\
\hline Rol / Relaciones & -No se observa & $\begin{array}{l}\text {-Interrupción en la relación de } \\
\text { apego madre e hijo. } \\
\text { - Dificultad en el desempeño del rol } \\
\text { materno. } \\
\text {-Interrupción de la lactancia } \\
\text { materna. }\end{array}$ \\
\hline Sexualidad & $\begin{array}{l}\text { •RNPT de sexo masculino } \\
\text {-Genitales de acuerdo a su } \\
\text { edad gestacional }\end{array}$ & •No se observa \\
\hline Afrontamiento / Tolerancia al estrés & -No se observa & $\begin{array}{l}\text { - El prematuro manifestó } \\
\text { irritabilidad. } \\
\text { - La madre manifestó ansiedad y } \\
\text { temor por la salud de su bebé y } \\
\text { frustración porno poder cuidarlo. }\end{array}$ \\
\hline Principios vitales & $\begin{array}{l}\text { - La mamá es católica, durante } \\
\text { su visita oraba por su bebé }\end{array}$ & •No se observa \\
\hline Seguridad/Protección & $\begin{array}{l}\text {-Se encontró en una servocuna } \\
\text { con técnica de nido acoplado. } \\
\text { Temperatura corporal: } 36.3^{\circ} \mathrm{C} \\
\text { - La piel se mantuvo limpia y } \\
\text { sin laceraciones }\end{array}$ & $\begin{array}{l}\text { - RNPT de } 36 \text { semanas de } \\
\text { gestación por Capurro. } \\
\text {-Recibió oxigenoterapia CPAP } \\
\text { burbuja. } \\
\text {-Presentó vía periférica. }\end{array}$ \\
\hline Confort & $\begin{array}{l}\text { •RNPT se encontró en una } \\
\text { servocuna }\end{array}$ & $\begin{array}{l}\text { - Prematuro mostró conductas } \\
\text { defensivas cuando se le realiza } \\
\text { algún procedimiento. }\end{array}$ \\
\hline Crecimiento/Desarrollo & $\begin{array}{l}\text {-RNPT AEG de } 36 \text { semanas de } \\
\text { gestación por Capurro } \\
\text {-Talla: } 45,5 \mathrm{~cm} \\
\text {-Circunferencia cefálica: } 32 \mathrm{~cm} \\
\text {-Circunferencia torácica: } 30 \mathrm{~cm}\end{array}$ & •Presentó BPN: $2280 \mathrm{~g}$ \\
\hline
\end{tabular}




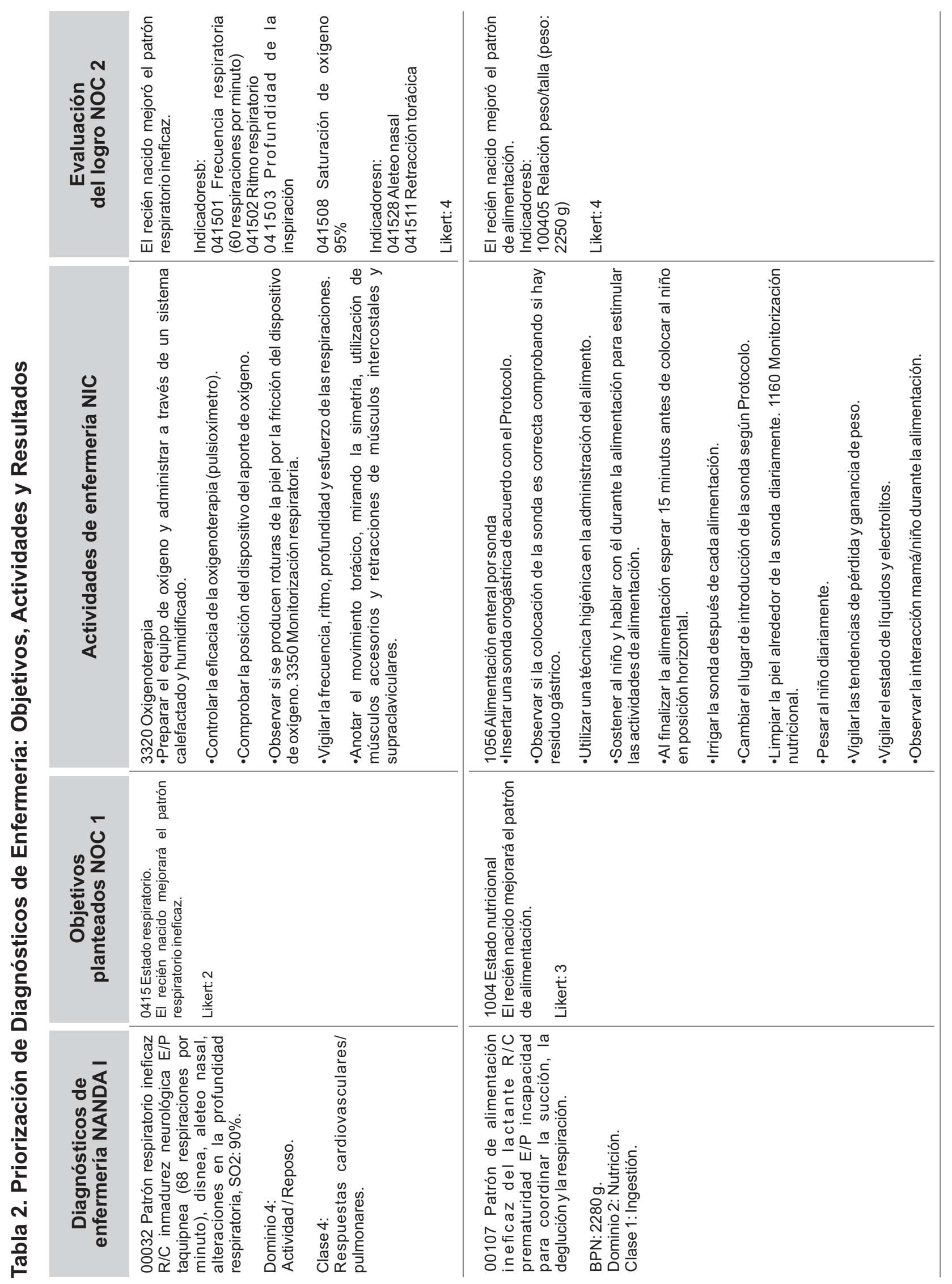




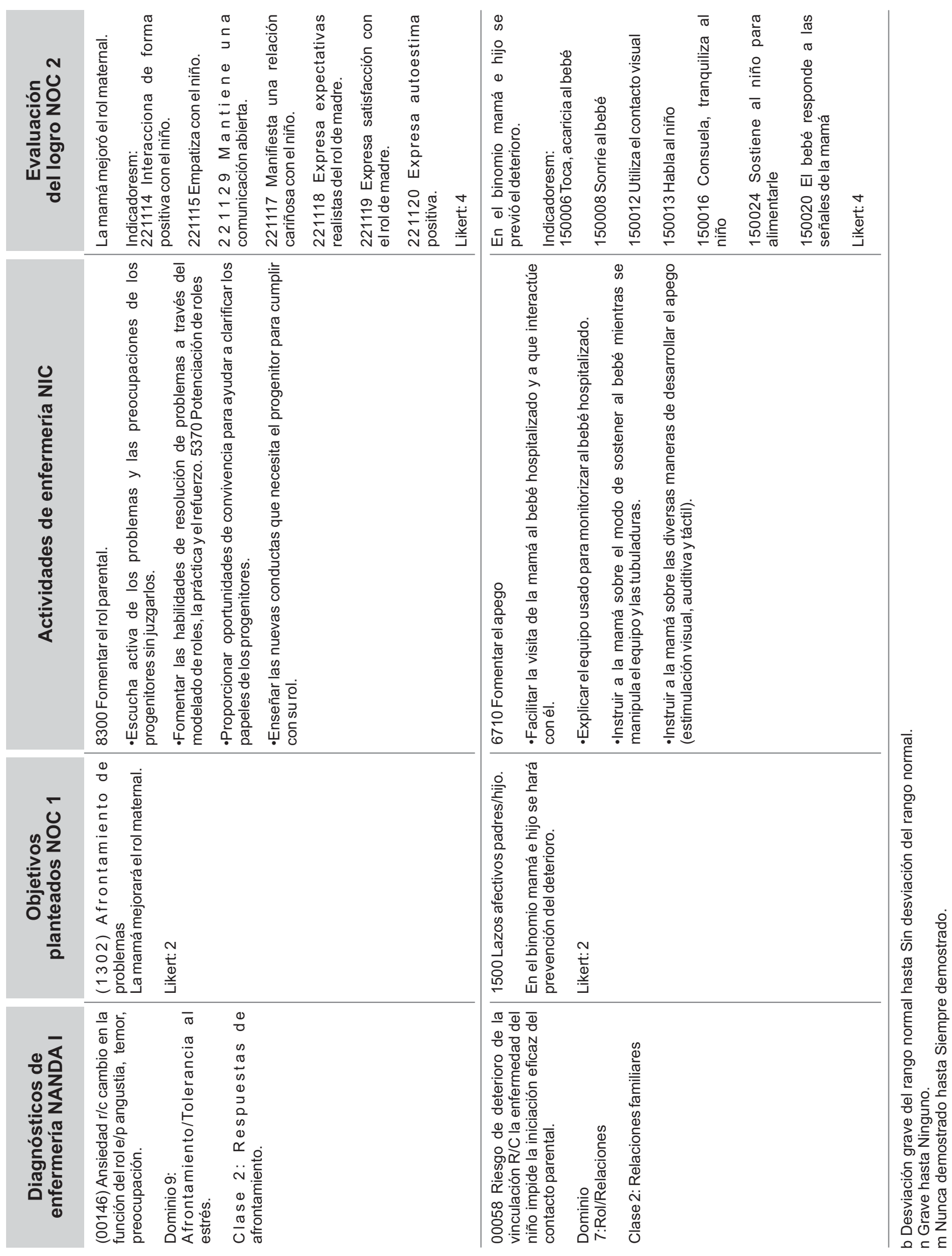




\section{DISCUSIÓN}

Ante la evaluación del plan de cuidados se concluye que se logró mejorar la eficacia de los patrones de respiración y alimentación disminuyendo así las complicaciones en la salud del recién nacido prematuro. Asimismo, se alcanzó fortalecer el vínculo materno filial logrando el bienestar psicológico y potenciando el rol de la mamá.

El análisis del caso puso en evidencia la eficacia de los cuidados técnicos neonatales que son brindados en la actualidad por el profesional de la salud especializado en Neonatología al recién nacido pretérmino de bajo peso con patología respiratoria. En un estudio reciente se demostró el beneficio de la ventilación no invasiva por CPAP de burbuja en donde el $88,17 \%$ de los pacientes que recibieron el tratamiento evolucionaron favorablemente (11). También en otra investigación se analizó la práctica de la alimentación enteral en recién nacidos pretérmino y señaló que existe la tendencia de que al menos se inicie una alimentación enteral mínima por sonda entre los primeros dos días de vida posnatal, para así evitar que el bebé padezca una deficiencia nutricional que le impida alcanzar un crecimiento ideal (12).

También el actuar de enfermería complementó los cuidados asistenciales con los afectivos. Se logró a través de una comunicación asertiva atenuar la ansiedad de la mamá ante la separación precoz con el recién nacido y se fomentó la participación activa de la mamá adaptando su rol durante el periodo de internación de su bebé. Asimismo, la estrategia de cuidado de acompañamiento materno facilitó el apego y fortaleció los lazos afectivos mejorando el vínculo entre la mamá y su hijo. Estos resultados se corroboran con investigaciones en donde se facilitó la estancia y participación de los familiares durante el periodo de internación de los pacientes pediátricos y neonatales. Con este acompañamiento se logró que los padres ejercieran un rol activo en el proceso de recuperación de sus hijos y disminuyera el estrés que produjo la separación $(13,14)$.

Las dificultades que se encontraron a la hora de poner en práctica el plan de cuidados fueron el escaso tiempo que se tuvo para orientar a la mamá en los cuidados y la inadecuada infraestructura de la unidad neonatal que no favoreció plenamente crear un ambiente de intimidad durante el contacto entre la mamá y el recién nacido.

Se recomienda que el personal de Enfermería durante su labor establezca una comunicación asertiva con los padres para conocer sus preocupaciones y/o expectativas. Elabore estrategias para facilitar el vínculo afectivo promoviendo la participación y colaboración de los padres acompañándolos en el proceso de apego para generar lazos emocionales satisfactorios entre padres e hijos. Ante la evaluación del plan de cuidados se concluye que se alcanzó fortalecer el vínculo materno filial logrando el bienestar psicológico y potenciando el rol de la mamá.

\section{REFERENCIAS BIBLIOGRÁFICAS}

1. Pérez J, Elorza D. Dificultad respiratoria en el recién nacido. An Pediatr Contin. 2003; 1(2): 57-66.

2. Méndez L, Martínez M, Bermúdez J. Unidad de Cuidados Intensivos Neonatales: Morbimortalidad en Recién Nacidos Prematuros. Acta Universitaria. 2007; 17(1): 46-51.

3. Oliveros M, Chirinos J, Costta R, Ticona M, Shimabuku R, Mestanza M, Manrique F. El recién nacido de muy bajo peso, proyecto multicéntrico. Revista Diagnóstico. 2005; 44(2): 54-59.
4. Martínez C, Romero G. Neonato pretérmino con dependencia en la necesidad de oxigenación y realización. Enfermería Universitaria. 2015; 12(3): 160-170.

5. Guerra M. Fortalecimiento del Vínculo Padres-Hijo a través del Proceso de Cuidado de Kristen Swanson. Revista Ciencia e Innovación en Salud. 2013; 1(2): 120125.

6. Porta R, Capdevila E. Un nuevo enfoque en la atención al recién nacido enfermo. El rol de los padres. Revista Iberoamericana de psicosomática. 2014; 109: 53-57.
7. Chupillón LE, Núnez AR. Rol materno en adolescentes primíparas y desarrollo del vínculo afectivo: servicio de neonatología, HRDLM, 2013. Paraninfo Digital. 2015; 4(22): 1-10.

8. Herdman TH, Kamitsuru S. NANDA International. Diagnósticos Enfermeros: Definiciones y Clasificación, 2015-2017. Barcelona: Elsevier; 2015.

9. Bulechek GM, Butcher HK, Dochterman JM, Wagner CM. Clasificación de Intervenciones de Enfermería (NIC). 6ta ed. Barcelona: Elsevier; 2014. 
10. Moorhead S, Johnson M, Maas ML, Swanson E. Clasificación de Resultados de Enfermería (NOC). 5ta ed. Barcelona: Elsevier; 2014.

11. Zemanate E, Bermúdez F, Manzi E, Ortiz K, Agudelo M. Experiencia con ventilación mecánica no invasiva en una unidad de cuidados intensivos pediátricos en Cali, Colombia. Acta Colombiana de Cuidado Intensivo. 2013; 13(4):237-243.
12. Udaeta E, Toussaint G, Pérez P, Carmona M. Alimentación enteral en el recién nacido pretérmino y de término con bajo peso: estado actual en México. Gac Méd Méx. 2005; 141(4): 283-290.

13. González MA. Los niños recién nacidos prematuros: nuevos actores sociales. Rev Hosp Mat Inf Ramón Sardá 2014; 33(3): 20-22.
14. Gallegos J, Reyes J, Silvan C. El neonato prematuro hospitalizado: significado para los padres de su participación en la Unidad Neonatal. Rev Latino-Am Enfermagem. 2013; 21(6): 1360-1366. 


\section{A care plan for premature with respiratory distress syndrome: strengthening the mother-newborn bond}

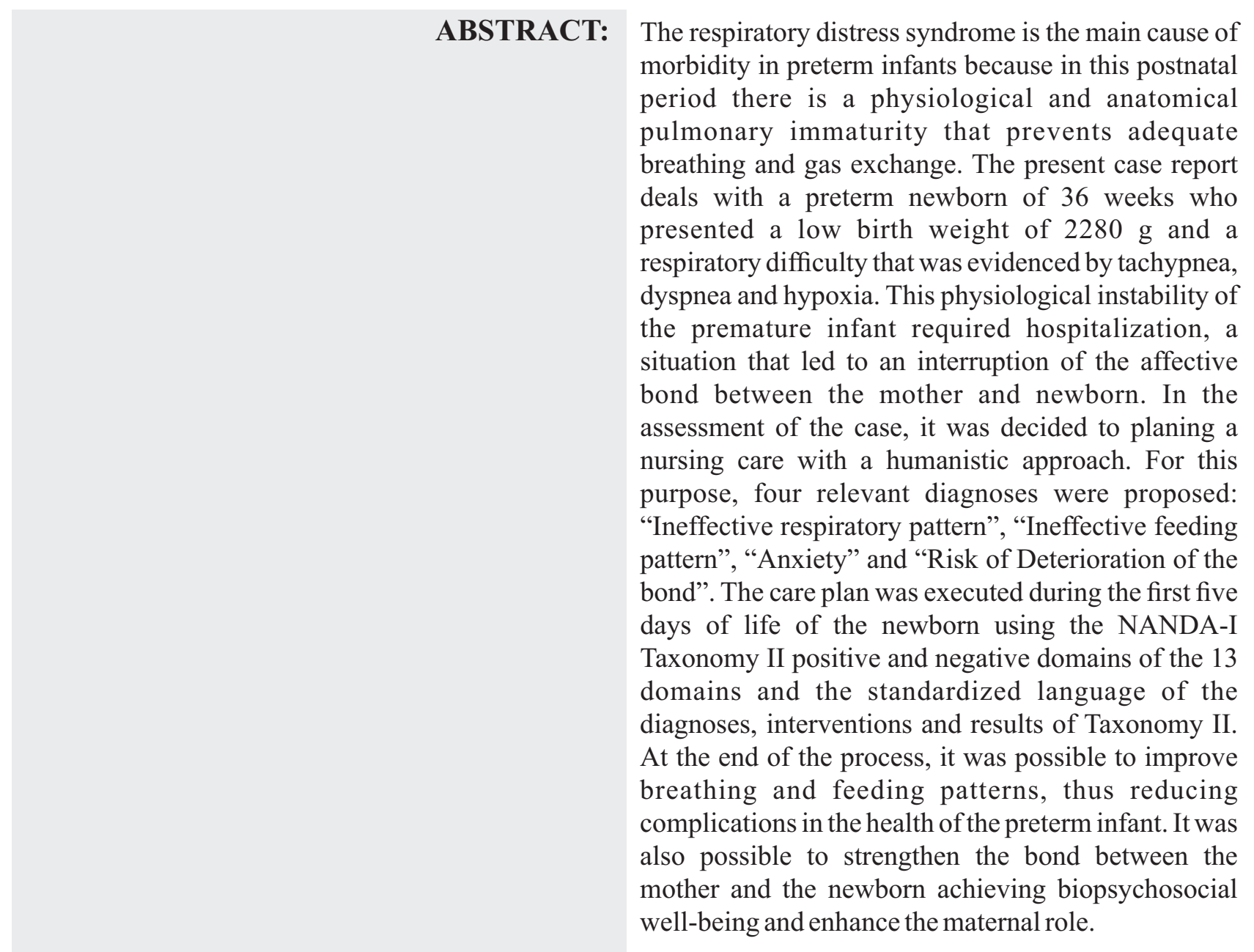

KEY WORDS: Nursing Care; Premature; Respiratory Distress Syndrome of the Newborn; Maternal bond. 